\title{
Téoros
}

Revue de recherche en tourisme

\section{Un système de cueillette et de traitement de données sur le tourisme à Montréal}

\section{Pierre Bellerose}

Volume 10, numéro 3, novembre 1991

Destination Montréal : d'hier à demain

URI : https://id.erudit.org/iderudit/1079203ar

DOI : https://doi.org/10.7202/1079203ar

Aller au sommaire du numéro

Éditeur(s)

Université du Québec à Montréal

ISSN

0712-8657 (imprimé)

1923-2705 (numérique)

Découvrir la revue

Citer cet article

Bellerose, P. (1991). Un système de cueillette et de traitement de données sur le tourisme à Montréal. Téoros, 10(3), 44-46. https://doi.org/10.7202/1079203ar d'utilisation que vous pouvez consulter en ligne. 


\section{Un système de cueillette et de traitement de données sur le tourisme à Montréal}

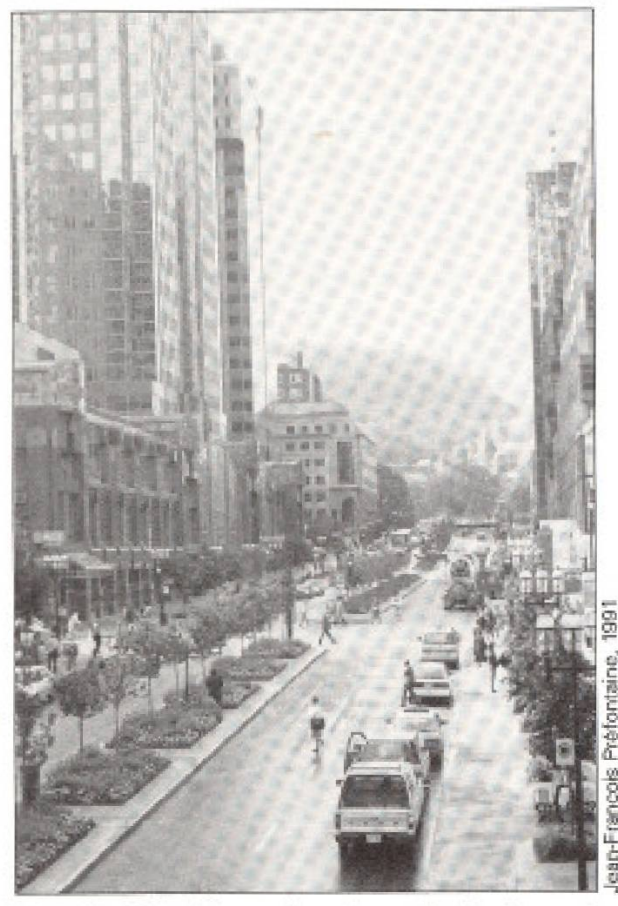

Le sysicme de cueillete of de traitement de donndes ste to pourisme a Montr fal permetora de mieict cerner notre rialite couristigue.
Monsieur Pierre Bellerose est directeur de la rechercheet du développement â l'Office des congrês et du tourisme du Grand Monteal (OCTGM).
L'importance économique des industries du tourisme et du voyage commence àêtre considérée comme un fait indéniable un peu partout dans le monde, et en particulier au niveau des États membres de l'OCDE.

À l'échelle canadienne, les gouvernements fédéral et provinciaux, notamment le ministère du Tourisme du Québec, commencent à se donner des orientations, des programmes et des outils qui illusirent assez bien la prise de conscience du fail que le tourismeest devenu une activité qui occupe une place majeure dans l' 6 conomie et se traduit de plus en plus par des investissements considérables aux retombées multiples, y compris en termes d'emplois.

Nul n'ignore plus que le développement de l'industrie touristique et la place grandissante des voyages tant d'affaires que d'agrément font que la concurrence entre les destinations est, et sera, de plus en plus féroce. Par ailleurs, la mondialisation de l'information, le développement de la publicité internationale et les échanges (politiques, commerciaux et culturels) entre les différents pays et villes rendent le marché touristique extrêmement vaste, complexe et compétitif.

Montréal n'échappe pas à ce contexte. Nombreux sont les décideurs politiques et économiques qui ont pris la mesure de $l^{\prime}$ environnement hautement concurrentiel qui éclaire des enjeux de taille, notamment en termes économiques.

A la suite du rapport Pour la relance du tourisme $e^{(1)}$ déposé en janvier 1988 , le Comité exécutif de la Ville de Montréal a adopté en juin 1988 un énoncé de politique qui souligne entre autres eque la Ville de Montréal entend désormais jouer pleinement son rôle et reconnaît de facto les opportunités exceptionnelles du développement économique que procurent les industries du tourismes(2).

Nombre de préoccupations ont été soulevées à l'occasion des études récentes réa- lisếes en matière touristique en ce qui concerne la région de Montréal.

Le document de janvier 1988 indiquait entre autres que: «L'industrie montréalaise du tourisme souffre du manque de données disponibles sur ses clientèles. Il y a un énorme effort de recherche à faire sur les clientèles, leurs origines, leurs motivations, leurs perceptionsde Montréal, leurs attentes, etc... de façon à mieux orienter la mise en marchéetaller chercher de façon spécifique de nouveaux sous-segments\% ${ }^{(9)}$.

Suite à ce constat, la Ville de Montréal décidait d'effectuer une étude de faisabilité en vue de la création d'un système de cueillette et de traitement de données sur le tourisme pour la région de Montréal ${ }^{(4)}$.

\section{Analyse des besoins}

\section{Inventaire des données existantes}

Une des premières opérations de l'étude a consisté à faire un bilan des données disponibles grâce à des rencontres avec une dizaine de producteurs de données au $\mathrm{Ca}$ nada. Bien sûr, le principal interlocuteur fut Statistique Canada qui a produitplusieurs enquêtes liées au tourisme.

De plus, d'autres organismes recensent des données liées au tourisme, soit le Bureau de la Statistique du Québec (hôtellerie), les firmes de consultants (Laventhol et Horwath) et les entreprises émettrices de cartes de crédit (American Express).

Globalement, il ressort de cette consultation auprès des producteurs qu'il existe un nombre important de données souvent disparates et peu connues mais qui laissent entrevoir un grand potentiel. Cette situation permetde confirmer les conclusions d'autres expériences au Canada (Edmonton) et d'affirmer qu'il existe actuellement suffisamment de matériel pour créer un systè̀me de cueillette et de traitement de données sur le tourisme à Montréal. C'est la chimie entre les ingrédients qui restait à définir. 


\section{Consultation auprès des utilisateurs}

Auprès de quelques quarante utilisateurs de données en tourisme, l'étude du Centre d'études du tourisme a constatế un intếrêt, voire un enthousiasme certain, pour un projet d'indicateurs de performance du tourisme à Montréal. Une des principales conclusions de cette consultation est que la très grande majorité des intervenants interviewés a indiqué que leur principal besoin se situait au niveau des donnces portant sur la région de Montréal.

\section{Les besoins en matière de données}

Il ressort des entrevues qu'en général, les données en tourisme servent à trois fins principales qui different selon 1 'interlocuteur. Ainsi, une majorité des personnes rencontróes utilise les données pour une finalité marketing. Il s'agit donc d'une étape d'un long processus qui vise à mieux connaître une clientèle (par exemple) avant d'accomplir certaines opérations de promotion. Les élé́ments qui reviennent le plus souvent sont les suivants:

- meilleure segmentation;

- flux des touristes étrangers;

- clientèles d'affaires:

- comparaison (avec les destinations concurrentes).

Pour d'autres, les données s'insèrent dans un processus de planification où l'on cherche à mieux positionner un produit ou la destination Montréal. Les planificateurs sont plus intéressés par des aspects comme les parts de marché, les tendances de l'offre et de la demande, les analyses prévisionnelles, les données de zones plus restreintes (centre-ville), la comparaison, etc.

Enfin, à peu près tous les intervenants rencontrés se sont dits intéressés par les ếtudes de retombées économiques, plus spécialement les gouvernements (fédéral, provincial, municipal) afin de justifier leurs budgets et leurs actions.

Le tableau 1 nous montre bien que les besoins de données sont différents d'un secteur à un autre. En effet, on constate des différences notables selon que l'intervenant touristique ocuvre dans le secteur de l'hébergement, du transport, de la restauration, des attractions, des activités professionnelles ou des influenceurs socioeconomiques.
La perception de la qualité des données actuellement disponibles

L'ensemble des intervenants consultés, sauf en de rares exceptions, se sont dits plus ou moins satisfaits ou insatisfaits des données actuellement disponibles sur le tourisme à Montreal, etcela pour les raisons suivantes:

- difficulté à quantifier le nombre de touristes venant à Montréal et leurs origines (surtout les non-canadiens);

- grave probleme de segmentation des données sur les clientèles venant à Montréal (surtout les clientèles provenant de l'étranger);

- manque de données importantes pour certains secteurs névralgiques (congres/salons, restauration, agences et grossistes);

- éparpillement des sources dedonnées;

- problèmes méthodologiques pour les données de certains secteurs (attractions);

- évaluation des dépenses des touristes;

- et finalement, pour certains intervenants, carences relatives aux investissements et à la fiscalité.

En fait, un seul secteur fait présentement l'unanimité, en termes de qualité des données générées, pour les rares personnes qui lesconnaissent: il s'agitdu transport aérien. Cette information statistique apparaît comme un indicateur indirect très important et qui n'a pas d'équivalent. On souligne cependant que ces données sont actuellement difficilement accessibles pour l'intervenant montréalais.

\section{Un projet pilote unique au Canada}

Compte tenu des besoins exprimés, des informations et indicateurs disponibles, des données potentielles, de l'expérience d'Edmonton et, bien sûr, du contexte montréalais, les auteurs de l'étude ont recommandé la mise en place d'un projet pilote de trois ans qui aurait comme objectif la mise sur pied d'un système de cueillette et de traitement de données.

Ce projet pilote devait se distinguer des expériences antérieures par son côté plus pragmatique, en tentant d'en arriver à des réalisations concrètes, tout en continuant le travail d'amelioration des indicateurs actuels. Le concept de projet pilote a comme objectif de faciliter le financement public et de permettre de prouver aux intervenants privés la faisabilité du système (ce qui permettra à long terme une implication financière de ces institutions).

\section{Une réalité depuis l'été 1991}

C'est à la suite des conclusions positives de l'étude de faisabilité du CET que la Ville de Montréal, de concert avec les gouvernements fédéral et provincial, ont pris la décision d'offrir aux intervenants touristiques montréalais des outils de qualité pour structurer et planifier leurs opérations marketing.

D'une durée de 30 mois, le projet des indicateurs de performance du tourisme a Montréal, qui a commencé ses opérations à l'étế 1991, est une première à l'échelle canadienne (ce projet est géré par l'OCTGM). II vise deux objectifs principaux:

a) La consolidation des indicateurs de performance actuellement utilisés en tourisme et la création de nouveaux indicateurs grâce à la mise en place des outils de recherche suivants:

\section{Enquête sur le comportement des} clienteles touristiques de Montréal:

Cette enquête qui sera entreprise auprès de 3000 touristes sur les sites d'attractions et dans les hôtels permettra de mieux définir les éléments qui composent les différents segments de touristes en visite à Montréal. Les résultats de cette ctude qui sera réalisé en 1992 seront intégrés à une base de données sur le tourisme. L'enquête sera confiée au Module de gestion et intervention touristiques de l'UQAM.

\section{Formation de comités-conseil:}

Le projet pilote prévoit la formation de comités-conseil qui évalueront la qualité des indicateurs de performance présentement utilisés au sein de l'industrie touristique montréalaise, en plus d'étudier la possibilité d'en développer de nouveaux. Trois comités seront formés: attractions, experts et investissements/emplois.

\section{Modele d'impact économique:}

Un modèle d'impact économique sera créé afin d'évaluer les retombées économiques régionales du tourisme et de démontrer leur importance par rapport aux autres secteurs de l'économie montréalaise. 
TABLEAU 1

Identification des principaux points d'intérêt exprimés par les interlocuteurs a Montréal pour la mise sur pied d'un système de cueillette et de traitement de données

\begin{tabular}{|c|c|c|c|c|c|}
\hline $\begin{array}{l}\text { Secteurs } \\
\text { Type }\end{array}$ & Het bergement & Transport & Attrastions & $\begin{array}{l}\text { Activites } \\
\text { professlonnelles }\end{array}$ & $\begin{array}{l}\text { Influences } \\
\text { soclo-tcononalques } \\
2\end{array}$ \\
\hline d'laformathon & & & & & \\
\hline \multirow{5}{*}{ Marketing } & $\begin{array}{l}\text { Nombre de touristes } \\
\text { utiliant les differonts } \\
\text { types d'hebergerment } \\
\text { (selon l" origine). }\end{array}$ & $\begin{array}{l}\text { Modo de irimsport } \\
\text { poer vemit i } \\
\text { Mantrésl. }\end{array}$ & $\begin{array}{l}\text { Develeppement diun } \\
\text { meilleur outil de } \\
\text { curllettr permettint } \\
\text { les comparaisons. }\end{array}$ & $\begin{array}{l}\text { Melleure segnentatian } \\
\text { des differentes clientules, }\end{array}$ & 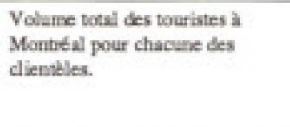 \\
\hline & $\begin{array}{l}\text { Grand besom its } \\
\text { segnentation plus }\end{array}$ & $\begin{array}{l}\text { Carantistiquas } \\
\text { des woynges selon }\end{array}$ & $\begin{array}{l}\text { Compontements } \\
\text { verce-touristiqus }\end{array}$ & $\begin{array}{l}\text { Compuraison des } \\
\text { differents regnerits avec }\end{array}$ & Identification des grouges-cibles \\
\hline & $\begin{array}{l}\text { pousuce des } \\
\text { elientitles. }\end{array}$ & $\begin{array}{l}\text { le mode do } \\
\text { transport. }\end{array}$ & das Montutalais. & Toroulh & $\begin{array}{l}\text { Analyses compuratives } \\
\text { (budpets de promotion, eta) }\end{array}$ \\
\hline & $\begin{array}{l}\text { Informations sur les } \\
\text { diffitentes diendiles } \\
\text { d'affairs. }\end{array}$ & $\begin{array}{l}\text { Riphartition des } \\
\text { diffitrents segmeats } \\
\text { selon l'origine }\end{array}$ & $\begin{array}{l}\text { Informations sur les } \\
\text { clientikles 6ungeres }\end{array}$ & & $\begin{array}{l}\text { Infomasion sur les cliemiles } \\
\text { ftringtos. }\end{array}$ \\
\hline & & & peographique. & & \\
\hline \multirow{6}{*}{ Manification } & $\begin{array}{l}\text { Evolurion de l'ofive } \\
\text { et de la demande. }\end{array}$ & $\begin{array}{l}\text { Tendaneeris) des: } \\
\text { (lix, }\end{array}$ & 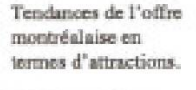 & $\begin{array}{l}\text { Pesceptìn de Moniréal } \\
\text { comme ville a congrés. }\end{array}$ & \multirow{2}{*}{ 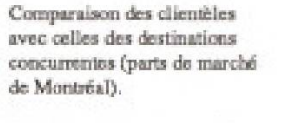 } \\
\hline & $\begin{array}{l}\text { Pevisions (aurtaut } \\
\text { cowert trime) }\end{array}$ & $\begin{array}{l}\text { Parts de marchó } \\
\text { des difféternts } \\
\text { types de trangpoet. }\end{array}$ & \multirow[t]{5}{*}{$\begin{array}{l}\text { Tendances de la } \\
\text { demande (sannut } \\
\text { intemationale) }\end{array}$} & $\begin{array}{l}\text { Evaluation de l'allio } \\
\text { (pettits, moyens ot gros } \\
\text { congrits). }\end{array}$ & \\
\hline & wones plus resteintes. & $\begin{array}{l}\text { Pofvisians court } \\
\text { et mogen knnes. }\end{array}$ & & Tendmees, & \multirow{4}{*}{ 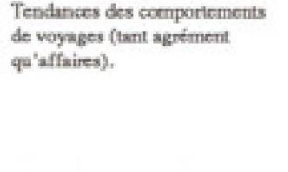 } \\
\hline & Camparaison Quefbed & & & Meilleure Evalustice de & \\
\hline & Toenton/Raston. & $\begin{array}{l}\text { Camporaison } \\
\text { (surtiout aves: }\end{array}$ & & $\begin{array}{l}\text { I'ofle pour wrades et } \\
\text { woyge-motivation. }\end{array}$ & \\
\hline & & $\begin{array}{l}\text { Torueb pour les } \\
\text { flux et atoports). }\end{array}$ & & & \\
\hline
\end{tabular}

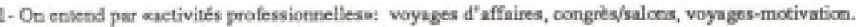

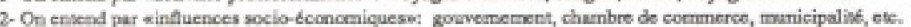

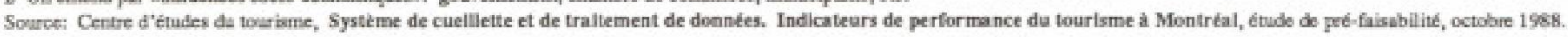

b- La création d'outils de diffusion efficaces qui maximiseront la transmission des indicateurs de performance:

\section{Base de données}

Une base de données informatisće centralisera un ensemble d'informations relatives au tourisme et diffusera efficacement et rapidement des renseignements souvent difficilement accessibles aux intervenants de l'industrie touristique montréalaise. Elle produira, à partir de données secondaires, des indicateurs de performance du tourisme. La création de la base de données sera supervisé par le Groupe DMR, spécialiste en gestion de l'information et des technologies.

\section{Bulletin statistique}

L'indicateur touristique est un des éléments-clés du projet pilote. Ce bulletin statistique sera publié trimestriellement et foumira les données essentielles à la compréhension globale de l'évolution du tou- risme à Montréal. On y retrouvera des indicateurs de performance des différents. secteurs d'activité touristique.

\section{Rapport annuel}

Un rapport annuel, beaucoup plus volumineux que la publication trimestrielle, sera produit à la fin de chaque année. Ce document présentera un bilan quantitatif et qualitatif de l'année écoulée, et cela tant globalement que par secteurs d'activités touristiques.

\section{Conclusion}

Il fait de plus en plus consensus que les données de base et les analyses appropriées sont des élements primordiaux dans le processus de décision. En effet, les actions dans le secteur du marketing et de la planification doivent $s$ 'appuyer sur des informations qui reflètent la réalité sinon des budgets importants peuventêtre mal utilisés. Grâce au soutien financier des gouvernements canadien, québécois et de la Ville de Montréal, la mise en place d'outils tels que ceux qui seront développés au cours de ce projet supportera la planification stratégique des activités touristiques montréalaises. Les impacts a long terme du projet se manifesteront par un meilleur positionnement de Montréal par rapport à ses principaux concurrents nord-américains et par des stratégies de marketing intelligentes et bien structurées. f

\section{Notes et références}

1) Rapport du Comite directeur de la demarehe de planification stratégique du développement du tourisme a Montrual, jarvier 19e9, $118 \mathrm{p}$.

2) Ennoné de politique de la Ville de Montréal en matière d'organisation de la promotion touristique, juin 1988, p. 12

3) Pour la relance du tourisme a Montréal, po, 83 B4.

4) Centre d'etudes du tourisme, Systame de cueil: lette ot de traitement de donnèes. Indicateurs de performanee du tourisme à Montréal l, étude de préfaisabilite, octobre $1988,90 \mathrm{p}$. 